\title{
Cloaked contact grids on solar cells by coordinate transformations: Designs and prototypes: supplementary material
}

Martin F. Schumann ${ }^{1,2,}$, , SAMUEl Wiesendanger $^{3}$, JAN Christoph Goldschmidt $^{4}$, Benedikt Bläsi ${ }^{4}$, Karsten BitTKau ${ }^{5}$, UlRich W. PAetzold ${ }^{5}$, Alexander Sprafke ${ }^{6}$, Ralf B. Wehrspohn ${ }^{6}$, Carsten Rockstuhl $^{2,7}$, and MARTIN WEGENER ${ }^{1,2}$

${ }^{1}$ Institute of Applied Physics, Karlsruhe Institute of Technology (KIT), 76128 Karlsruhe, Germany

${ }^{2}$ Institute of Nanotechnology, Karlsruhe Institute of Technology (KIT), 76021 Karlsruhe, Germany

${ }^{3}$ Institute of Condensed Matter Theory and Solid State Optics, Friedrich-Schiller-Universität Jena, 07743 Jena, Germany

${ }^{4}$ Fraunhofer Institute for Solar Energy Systems ISE, Heidenhofstraße 2, 79110 Freiburg, Germany

${ }^{5}$ Institut für Energie- und Klimaforschung (IEK-5), Forschungszentrum Jülich GmbH, 52425 Jülich, Germany

${ }^{6}$ Institute of Physics, Martin-Luther-University Halle-Wittenberg, 06120 Halle, Germany

${ }^{7}$ Institute of Theoretical Solid-State Physics, Karlsruhe Institute of Technology (KIT), 76128 Karlsruhe, Germany

*Corresponding author: martin.schumann@kit.edu

Published 25 September 2015

This document provides supplementary information to "Cloaked contacts on solar cells designed by coordinate transformations," http://dx.doi.org/10.1364/optica.2.000850. (C) 2015 Optical Society of America

http://dx.doi.org/10.1364/optica.2.000850.s001

\section{TRUNCATING THE REFRACTIVE INDEX MAP}

Generally, the mapping function for mapping a half-plane to a polygon with $m$ corners can be written as [1]

$$
f(u)=C_{1}+C_{2} \int_{u_{0}}^{u} \prod_{i=1}^{m}\left(\tilde{u}-u_{i}\right)^{\varphi_{i} / \pi-1} \mathrm{~d} \tilde{u},
$$

where $\varphi_{i}$ denotes the angle enclosed by the $i^{\text {th }}$ corner of the polygon and $C_{1}$ and $C_{2}$ are complex constants. For our application, we choose a polygon with three corners $v_{i}$. While corners $v_{1}$ and $v_{2}$ are finite, the third corner lies at infinity. The complex constants $C_{1}$ and $C_{2}$ are chosen such that the prevertices $u_{1}$ and $u_{2}$ are mapped to the two finite polygon corners, i.e., $v_{1}=f\left(u_{1}\right)$ and $v_{2}=f\left(u_{2}\right)$. The prevertices are bound to be real, i.e., $\operatorname{Im}\left(u_{1}\right)=\operatorname{Im}\left(u_{2}\right)=0$. Further restrictions apply for the polygon corners, namely $\operatorname{Im}\left(v_{1}\right)=0$ and $\operatorname{Im}\left(v_{2}\right)=R_{1}$. From the mapping function $f(u)$ we can derive the refractive index distribution,

$$
n(u)=n_{0}\left|\frac{\mathrm{d} f}{\mathrm{~d} u}\right|^{-1}=n_{0}\left|\prod_{i=1}^{m}\left(u-u_{i}\right)^{1-\varphi_{i} / \pi}\right|,
$$

where $n_{0}$ denotes the refractive index of the untransformed space. Thus, a total of five real parameters has to be chosen to obtain the refractive index distribution. We pick the parameter set

$$
\begin{aligned}
& n_{0}=1.5 \\
& u_{1}=\operatorname{Re}\left(v_{1}\right)=-1.5 R_{1} \\
& u_{2}=\operatorname{Re}\left(v_{2}\right)=0
\end{aligned}
$$

resulting in a refractive index distribution centered around $n \approx 1.3$ (see Figure $1(\mathrm{~b})$ in the main text).

We spatially crop the refractive index distribution to a finite region, $A$. The edges of $A$ are chosen to have a minimal distance of $5 R_{1}$ to either of the polygon vertices, $v_{1}$ and $v_{2}$ (see Figure $\mathrm{S} 1$ ). Like this, high refractive index gradients are constrained to the center of the refractive index distribution. We have furthermore optimized $\operatorname{Re}\left(v_{1}\right)$ such that the refractive index gradient integrated along the right edge of $A$ is minimal. This means that there is a relatively constant refractive index at the interface between graded-index region and solar cell substrate. To end up with the refractive index distribution depicted in Figure 1(b) of 


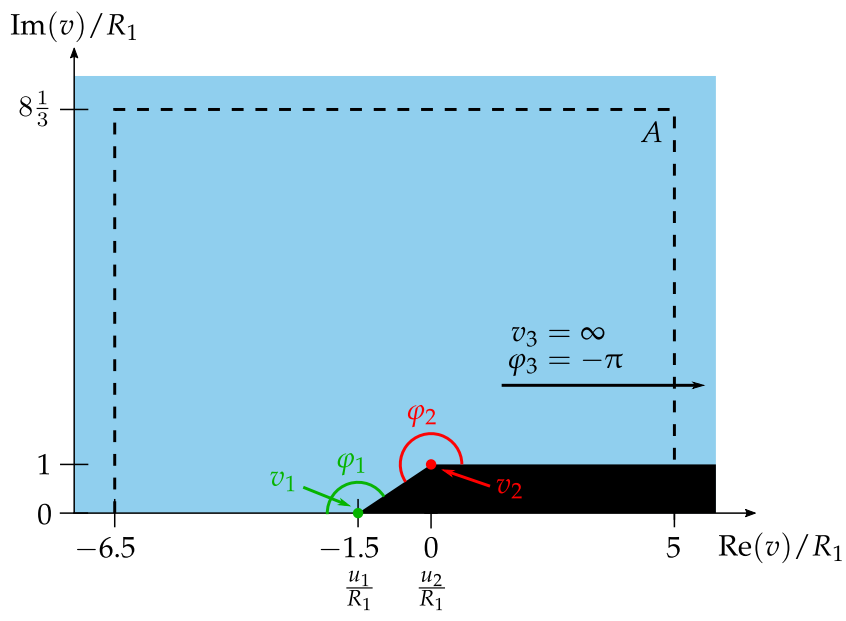

Fig. S1. Illustration of the polygon chosen for the SchwarzChristoffel transformation. While the polygon corners $v_{1}$ and $v_{2}$ are finite, $v_{3}$ is set to infinity. We crop the spatially infinitely extended resulting refractive index map to a finite region, $A$ (dashed line). The complete index distribution (Figure 1(b) of the main text) is obtained by rotating the cropped region by $-90^{\circ}$ and mirroring it to the left.

the main text, we rotate the spatially cropped refractive index by $-90^{\circ}$ and mirror it to the left.

Obviously, the refractive index distribution as derived from the mapping function exhibits singularities at $u=u_{i}$ (see Equation (S1)). While for positive exponents the refractive index becomes zero, it diverges in the case of negative exponents. Since only refractive index values $n \in[1.0,1.5]$ are amenable to our fabrication scheme, we truncate the values of the refractive index distribution, $n(u)$, to this interval. Ray-tracing results through the truncated index distribution at perpendicular incidence are depicted in Figure S2 (top). It can be seen that some rays enter the forbidden region above the contact, in contrast to the un-truncated case (Figure 1(b) of the main text), where light rays would propagate along the transformed coordinate lines. This leads to a slightly decreased performance of the truncated distribution at perpendicular incidence. Obviously, the choice of material for the forbidden region can influence the performance of the invisibility structure. This is especially visible at oblique incidence (Figure S2, bottom), where the hollow version performs much better than the filled version of the graded-index structure due to total internal reflection at the air core (see also annual average improvements given in Table S1).

\section{DERIVATION OF THE FREE-FORM SURFACE FUNC- TION}

In order to derive the free-form surface function $y(x)$ (for $x>0$ ), we substitute $x^{\prime}$ in $\tan (\alpha)=\frac{x^{\prime}-x}{y}$ by Equation (2) in the main text. Applying Snell's law, we find an implicit equation for the surface inclination angle

$$
\beta(x)=\arctan \left(\frac{R_{1}}{y(x)}\left(1-\frac{x}{R_{2}}\right)\right) .
$$

Since $\tan (\beta)=\mathrm{d} y / \mathrm{d} x$, the surface function $y(x)$ can be expressed as

$$
y(x)=y(0)+\int_{0}^{x} \tan (\beta(\tilde{x})) \mathrm{d} \tilde{x} .
$$
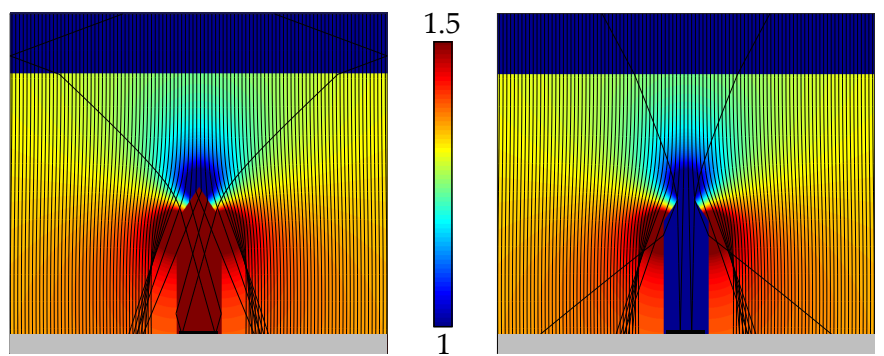

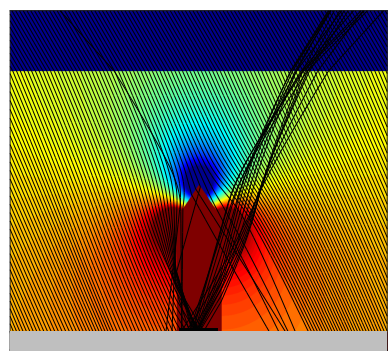

(a)

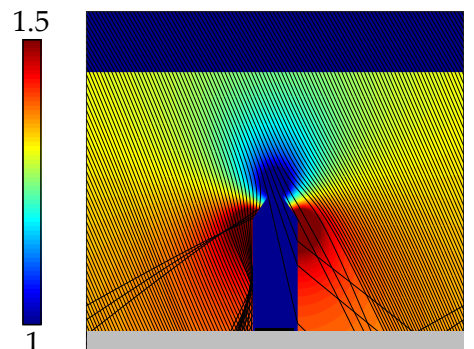

(b)
Fig. S2. Ray-tracing simulations through the truncated refractive index distribution (color-coded) with exemplary rays (black lines) at perpendicular incidence (top) and at oblique incidence (bottom). The forbidden region is filled with full material (a) and air (b), respectively. In any case, rays enter the forbidden region and hit the contact (black bar), i.e., cloaking is not ideal. While for perpendicular incidence, filled and hollow version offer comparable performance, the graded-index structure with air core outperforms the filled variant due to total internal reflection at the air core. For better clarity, partially reflected rays are not drawn. The rays are traveling fully in the $x y$-plane, i.e., $\psi_{2}=0^{\circ}$. 
We substitute $y(x)$ in Equation (S2) by Equation (S3), leading to an implicit equation for the surface inclination angle $\beta(x)$,

$$
\frac{R_{1}\left(1-\frac{x}{R_{2}}\right)}{\tan \left(\beta(x)-\arcsin \left(\frac{\sin (\beta(x))}{n}\right)\right)}-y(0)=\int_{0}^{x} \tan (\beta(\tilde{x})) \mathrm{d} \tilde{x}
$$

This equation can either be solved numerically or approximated analytically for the case of small angles.

\section{A. Numerical Solution}

For the numerical solution of Equation (S4) we choose a recursive approach. We split the interval from $x_{1}=0$ to $x_{N+1}=R_{2}$ into $N$ equidistant sub-intervals of length $\Delta x$. Thus, the starting position of the $i^{\text {th }}$ interval is at $x_{i}=(i-1) \cdot \Delta x$, with $i=1 . . N$. Using this discretization, we can rewrite Equation (S4) for position $x=x_{i}$ as

$$
\frac{R_{1}\left(1-\frac{x_{i}}{R_{2}}\right)}{\tan \left(\beta\left(x_{i}\right)-\arcsin \left(\frac{\sin \left(\beta\left(x_{i}\right)\right)}{n}\right)\right)}-y(0)=I_{i},
$$

where the right hand side is the integral

$$
I_{i}=\int_{0}^{x_{i}} \tan (\beta(\tilde{x})) \mathrm{d} \tilde{x} .
$$

In order to calculate the integral, we split it according to the recursion relation

$$
I_{i}=I_{i-1}+\int_{x_{i-1}}^{x_{i}} \tan (\beta(\tilde{x})) \mathrm{d} \tilde{x} \approx I_{i-1}+\tan \left(\beta\left(x_{i-1}\right)\right) \cdot \Delta x .
$$

Substituting $I_{i}$ back into Equation (S5) we obtain a recursion relation for $\beta\left(x_{i}\right)$ which can be evaluated numerically starting at $i=2$. The starting value $\beta\left(x_{1}=0\right)$ is computed using Equation (S5), where the right hand side $I_{1}$ trivially vanishes. Once the surface tilt angle $\beta\left(x_{i}\right)$ and the integral $I_{i}$ are calculated for a given position $x_{i}$ using the recursion formulae, they can be translated to a discretized surface height $y\left(x_{i}\right)$ by using Equation (S3) in its discretized formulation, i.e., $y\left(x_{i}\right)=y(0)+I_{i}$.

\section{B. Analytical Approximation}

If the angles occurring in Equation (S4) are assumed to be small, i.e., $\alpha \ll \pi / 2$ and $\beta \ll \pi / 2$, the trigonometric functions in Equation (S4) can be substituted by their respective arguments. Furthermore we use Equation (S3) in its differential formulation to obtain a differential equation for the surface function

$$
y^{\prime}(x) \cdot y(x)=\frac{R_{1}}{1-\frac{1}{n}}\left(1-\frac{x}{R_{2}}\right),
$$

which can be solved analytically. Thus, for small angles, we obtain the surface function

$$
y(x)=\sqrt{y^{2}(0)+\frac{R_{1}}{1-1 / n}\left(2 x-\frac{x^{2}}{R_{2}}\right)} .
$$

Figure S3 compares the free-form surface function obtained by the analytical approximation and the numerical solution for a typical set of parameters. It has to be noted that, despite the good agreement of the analytical approximation with the numerical solution, we have used the numerical solution for all fabricated structures and ray tracing simulations shown in this Letter.

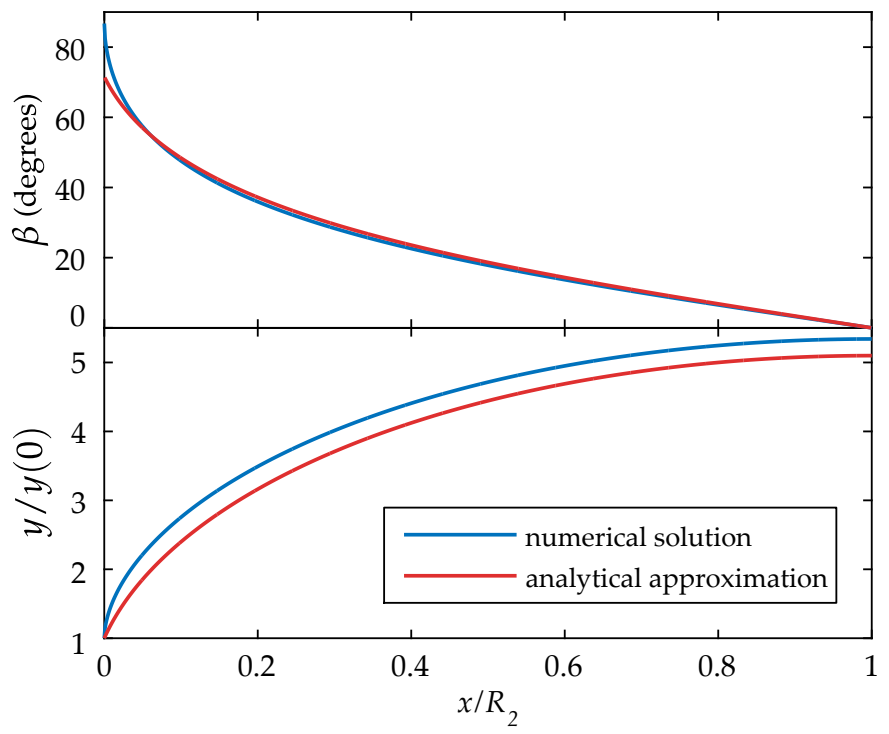

Fig. S3. Comparison of the numerical solution for the local surface inclination angle $\beta(x)$ (top) and the surface function $y(x)$ (bottom) of the free-form structure with its respective analytical approximations. For the curves shown we assume a periodic arrangement of contacts with width $w=R_{1} / 0.6$, an areal filling fraction of $10 \%$, and a constituent material with refractive index $n=1.5$. Hence, the geometric parameters are chosen $R_{2} / w=5$ and $y(0) / R_{1}=1$, as for the fabricated samples and the ray tracing simulations. In this case, the analytical approximation resembles the numerical solution very well, despite slight deviations for $x \lesssim 0.05 R_{2}$.

\section{Parameter Choice}

Once filling fraction $f$ and width $w$ of the contact, as well as the refractive index $n$ of the constituent material of the free-form surface, are given, there are three remaining parameters, $R_{1}, R_{2}$ and $y(0)$, that define the geometry of the free-form surface. They, however, cannot be chosen completely freely, but underlie the following physical limitations.

Firstly, for the free-form surface to provide perfect cloaking under perpendicular incidence, $R_{1}$ must be larger than $w / 2$ and smaller than the period with which the contact grid lines are arranged, such that

$$
\frac{R_{1}}{w} \in\left[\frac{1}{2}, \frac{1}{2 f}\right]
$$

While for $R_{2}$ the same upper limit applies, the lower limit is given by the choice of $R_{1}$ :

$$
\frac{R_{2}}{w} \in\left[\frac{R_{1}}{w}, \frac{1}{2 f}\right] .
$$

The choice of the second parameter, $y(0)$, is limited by the fact that the local surface inclination angle $\beta(x)$ must be smaller or equal to $\pi / 2$ for all $x \in[0, w /(2 f)]$. Since $\beta(x)$ decreases monotonically (see Figure S3), this effectively imposes a limit on $\beta(x=0)$, i.e., $\beta(0) \leq \pi / 2$. This is translated to a limitation for the choice of $y(0) / R_{1}$ by evaluating Equation (S4) at $x=0$ and $\beta(0)=\pi / 2$, leading to a lower limit for the choice of $y(0) / R_{1}$. There is no physical reason for an upper limit for the parameter, such that 


$$
\frac{y(0)}{R_{1}} \geq\left[\tan \left(\frac{\pi}{2}-\arcsin \left(\frac{1}{n}\right)\right)\right]^{-1}
$$

is the only limitation regarding the choice of $y(0) / R_{1}$.

We compute the annual average of the relative improvement (see Section 4) including all reflections for different choices of parameters to find the optimum set of $y(0), R_{1}$, and $R_{2}$ for given filling fraction $f=0.1$, refractive index $n=1.5$, and contact width $w$. From the results of the parameter sweep it is evident that the highest annual average is obtained when $R_{2} / w$ is maximal while $R_{1} / w$ and $y(0) / w$ are minimal, i.e.,

$$
\begin{aligned}
& \frac{R_{1}}{w}=\frac{1}{2}, \\
& \frac{R_{2}}{w}=\frac{1}{2 f}=5, \\
& \frac{y_{0}}{w}=\frac{1}{2}\left[\tan \left(\frac{\pi}{2}-\arcsin \left(\frac{1}{n}\right)\right)\right]^{-1} \approx 0.45 .
\end{aligned}
$$

For practical reasons, however, we choose slightly modified parameters for the fabricated structures. If one chooses the smallest possible value for $R_{1} / w$, small misalignments between the prefabricated model contact and the free-form surface already lead to degraded performance. Thus, we choose the slightly higher $R_{1} / w=0.6$ instead of the optimum value. Furthermore, reducing $y_{0} / w$ to the smallest possible value leads to a surface inclination angle of $90^{\circ}$ at $x=0$, and thus a very sharp slit, which is not easy to manufacture using DLW. For this reason, we choose $y_{0} / w=0.6$ for the fabricated structures. Ray-tracing simulations ignoring partial reflections show ideal performance $\langle\zeta\rangle=0.11$ for structures with both the optimum and the modified set of parameters. Including partial reflections, the structure with the modified parameter set exhibits an annual average relative improvement $\left\langle\zeta_{\text {flat }}^{\prime}\right\rangle$ less than $0.5 \%$ lower than the structure with the optimum parameter set.

\section{DIRECT LASER WRITING}

The proof-of-principle contact invisibility structures are fabricated using a commercial direct laser writing (DLW) machine (Photonic Professional GT, nanoscribe GmbH, EggensteinLeopoldshafen, Germany). We employ so-called Dip-In writing, i.e., the focusing objective is immersed in the photoresist (IP-Dip, nanoscribe GmbH, Eggenstein-Leopoldshafen, Germany). For both types of invisibility structures, the laser focus is scanned within the photoresist by means of a galvoscanner, enabling writing speeds of $30 \mathrm{~mm} \mathrm{~s}^{-1}$ and $50 \mathrm{~mm} \mathrm{~s}^{-1}$ for the graded-index and the free-form surface structures, respectively. Adhesion of the polymer structures to the silicon substrate is enhanced by treatment of the substrate with (3-Methacryloxypropyl)trimethoxysilane [2] prior to the DLW step.

In order to realize the graded-index structure, we use the socalled $N$-rod technique [3], where every rod along a predefined axis of the woodpile consists of $N$ individual lines with a spacing of $0.1 \mu \mathrm{m}$ in between. By adjusting $N$, the local volume filling fraction of polymer vs. air is set up to match the effective refractive index given by the index distribution shown in Figure 1(b) in the main text. The lateral lattice constant of the woodpile is designed as $a_{0}=900 \mathrm{~nm}$. Due to post-writing shrinkage of the polymer, the actual rod distance is slightly lower at $a=800 \mathrm{~nm}$. Along the axial direction, the overall structure consists of 107.25 unit cells (429 layers) stacked with a design lattice constant of $c_{0}=\sqrt{2} a_{0}=1.27 \mu \mathrm{m}$. Again, shrinkage reduces the total height of the graded-index device from the theoretical value of $136 \mu \mathrm{m}$ to an actual value of $132 \mu \mathrm{m}$. Below the actual graded-index structure, a $7 \mu \mathrm{m}$ high solid polymer block is written to improve adhesion of the device to the silicon substrate. In terms of writing times, a graded-index structure sized as the one shown in Figure 3(a) of the main text lies near $1 \mathrm{~h}$.

For the free-form surface structures, we write only the shell of the structures with DLW, leaving the interior unexposed. After washing away the unexposed resist outside of the written structure with a commercial photoresist developer (mr-Dev 600, micro resist technology $\mathrm{GmbH}$, Berlin, Germany), we expose the remaining unexposed resist with a $1.2 \mathrm{~mW}$ high-power LED emitting at a wavelength of $405 \mathrm{~nm}$. Using this core-shell writing approach the writing time reduces to below $10 \mathrm{~min}$ for a typically-sized free-form structure (see Figure 3(b) of the main text).

\section{COMPUTATION OF THE ANNUAL AVERAGE IM- PROVEMENT}

We define the annual average relative improvement $\langle\zeta\rangle$ obtained by the invisibility structure as

$$
\begin{aligned}
\langle\zeta\rangle+1 & =\frac{\text { energy deposited on active area with cloak }}{\text { energy deposited on active area without cloak }} \\
& =\frac{\int_{\text {year }}(\zeta(t)+1) T_{\mathrm{aS}}(t) I_{\mathrm{dir}}(t)+\overline{T_{\text {cloak }}} 1 I_{\text {diff }}(t) \mathrm{d} t}{\int_{\text {year }} T_{\mathrm{aS}}(t) I_{\text {dir }}(t)+\overline{T_{\mathrm{aS}}} I_{\text {diff }}(t) \mathrm{d} t} .
\end{aligned}
$$

Here, $\zeta(t)$ denotes the relative improvement compared to a silicon solar cell without any cloaking or anti-reflective measures, but with the same areal filling fraction of the contact (see Equation (S9)). $I_{\text {dir }}(t)$ is the direct irradiance on the solar cell, and $T_{\mathrm{aS}}(t)$ is the transmittance between air $(n=1)$ and Silicon $(n=4)$ calculated using the Fresnel equations for unpolarized light. $I_{\text {diff }}(t)$ denotes the diffuse irradiance on the cell, with $\overline{T_{\mathrm{aS}}}$ being the angle-averaged Fresnel transmission from air to silicon and $\overline{T_{\text {cloak }}}$ being the angle-averaged transmission from air to silicon with the cloaking structure in place. Isotropic illumination is assumed, i.e.,

$$
\begin{aligned}
\overline{T_{\mathrm{aS}}} & =\int_{0}^{\pi / 2} T_{\mathrm{aS}}(\vartheta) \sin \vartheta \mathrm{d} \vartheta \\
\overline{T_{\text {cloak }}} & =\frac{1}{2 \pi} \int_{0}^{2 \pi} \int_{0}^{\pi / 2}(\zeta(\vartheta, \varphi)+1) T_{\mathrm{aS}}(\vartheta) \sin \vartheta \mathrm{d} \vartheta \mathrm{d} \varphi
\end{aligned}
$$

To find the time-dependent angle of incidence $\Theta(t)$ needed to calculate the time-dependent quantities in Equation (S8), we compute the sun azimuth $\alpha_{\mathrm{S}}(t)$ and the sun height $\gamma_{\mathrm{s}}(t)$ (Figure S4). These quantities are calculated according to DIN 5034, part 2 (Ref. [4]) for Karlsruhe conditions (latitude $49.007^{\circ} \mathrm{N}$, longitude $8.404^{\circ} \mathrm{E}$, Central European Time (CET)). Any type of wavelength-dependence is ignored.

We consider a solar cell that is tilted towards the horizontal by inclination angle $\gamma_{c}=36^{\circ}$ and rotated around the vertical axis by azimuthal angle $\alpha_{\mathrm{c}}=6^{\circ}$ (Figure S4). This is the optimum orientation for a non-tracking solar cell installed in Karlsruhe [5]. To simplify calculations, we introduce three vectors with unity length: the normal vector $\vec{n}$, the vector $\vec{s}$ pointing towards 
the sun, and vector $\vec{e}$, which points up the slope of the solar cell along its edge (see Figure S4). Like this, the angle of incidence $\Theta(t)$ on the tilted solar cell becomes

$$
\Theta(t)=\arccos (\vec{s}(t) \cdot \vec{n}) .
$$

The time-dependent irradiances on the tilted plane, $I_{\text {dir }}(t)$ and

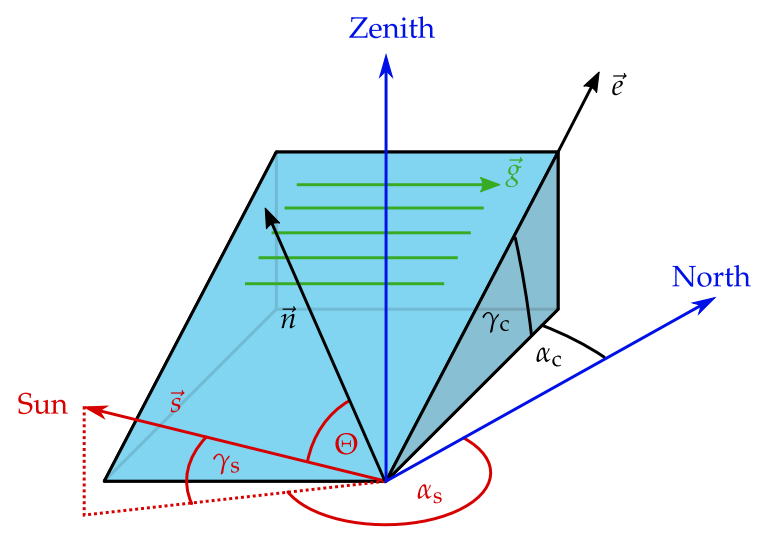

Fig. S4. Illustration of the orientation of a tilted solar cell with contact grid lines (green). It has inclination $\gamma_{c}$, azimuth $\alpha_{c}$, and normal vector $\vec{n}$. Vector $\vec{e}$ points up the slope of the cell, along its edge. The position of the sun is represented by the sun height $\gamma_{\mathrm{s}}$ and the sun azimuth $\alpha_{\mathrm{s}}$, or, alternatively, by vector $\vec{s}$ pointing towards the sun. The incidence angle $\Theta$ is the angle between $\vec{s}$ and $\vec{n}$. The contact grid lines are oriented along direction $\vec{g}$, perpendicular to the $(\vec{n}, \vec{e})$-plane. Despite the visually different length of the vectors, all vectors are considered normalized to unity length.

$I_{\text {diff }}(t)$ are derived from the irradiance data for the horizontal, $I_{\text {dir,hor }}(t)$ and $I_{\text {diff,hor }}(t)$, respectively:

$$
\begin{aligned}
I_{\text {dir }}(t) & =I_{\text {dir,hor }}(t) \cdot \frac{\cos (\Theta(t))}{\sin \left(\gamma_{\mathrm{s}}\right)} \\
I_{\text {diff }}(t) & =I_{\text {diff,hor }}(t) \cdot \frac{1}{2}\left(1+\cos \gamma_{\mathrm{c}}\right) .
\end{aligned}
$$

The irradiance data on the horizontal plane for Karlsruhe is taken from the CM-SAF PVGIS database $[5,6]$.

We decompose $\Theta(t)$ into the component $\psi_{1}(t)$ lying in the $(\vec{n}, \vec{e})$ plane and the component $\psi_{2}(t)$ in the $(\vec{n}, \vec{g})$-plane. We then substitute $\zeta(t)$ in Equation (S8) by the values for $\zeta\left(\psi_{1}(t), \psi_{2}(t)\right)$ obtained in ray tracing simulations and evaluate the integrands in Equation (S8) throughout one calendar year.

\section{RAY TRACING SIMULATIONS}

\section{A. Method}

The ray-tracing results shown in this Letter are obtained using a home-built ray-tracing software. While the rays are traced in three dimensions, the refractive index distribution is assumed to be invariant along the third dimension, i.e., $n=n(x, y)$. Here, the coordinate system is chosen such that the directional vectors shown in Figure S5 coincide with the coordinate axes, i.e.,

$$
\vec{e}=\left(\begin{array}{l}
1 \\
0 \\
0
\end{array}\right), \vec{n}=\left(\begin{array}{l}
0 \\
1 \\
0
\end{array}\right), \vec{g}=\left(\begin{array}{l}
0 \\
0 \\
1
\end{array}\right) .
$$

The width of the simulation region is equal to one period of the arrangement of contact fingers. Periodic boundary conditions are imposed along this direction, i.e., rays leaving the simulation region at the right end re-enter it at its left end and vice versa. We launch $N_{0}=1000$ equally spaced rays at the top of the simulation region and trace them either through the spatially truncated index distribution as depicted in Figure 1(b) of the main text or through a free-form structure whose surface obeys Equation (S4). The launch direction of the rays is given by the angles $\psi_{1}$ and $\psi_{2}$. Both are measured towards the $y$-axis. $\psi_{1}$ is the component lying in the $x y$-plane, and $\psi_{2}$ is the out-of-plane angle, measured in the $y z$-plane. Using these definitions, normal incidence is characterized by $\psi_{1}=\psi_{2}=0$.

For the free-form surface design, ray-tracing is straight forward: We calculate the intersection points of the rays with interfaces between regions with different refractive indices and apply Snell's law. This procedure is repeated until the rays leave the simulation region at the top or the bottom.

In the case of the graded-index structure, the rays are traced using a Newtonian approach [7], which is applicable for a continuously varying refractive index. At locations where discrete index jumps occur (e.g. at the surface of the spatially truncated index distribution), we replace the Newtonian method by Snell's law to correctly describe the transmitted ray.

For better clarity, for both situations partial reflections $(R \neq 1)$ are ignored by default. Total reflections $(R=1)$ like internal reflections or reflections off the contact, however, are accounted for. With this approach, the relative improvement $\zeta$ can be calculated from the number of rays $N$ transmitted into the silicon substrate as

$$
\zeta=\frac{N}{N_{0}(1-f)}-1,
$$

where $f$ denotes the areal filling fraction of the contact. Thus, positive values for $\zeta$ imply that more rays reach the silicon substrate with invisibility structure than without it. Since the maximum possible number of rays reaching the substrate is $N_{\max }=N_{0}$, the maximum possible improvement becomes

$$
\zeta_{\max }=\frac{f}{1-f}
$$

\section{B. Including Fresnel Reflections}

Ignoring partial reflections leads to results that are intuitive and straight-forward to interpret. However, in order to assess the performance of the invisibility structures in a more realistic way, partial reflections must be included. This is done by assigning a power value (initial value $P_{0}$ ) to every ray. Upon hitting an interface, the rays (with power $P_{\mathrm{i}}$ ) are split into a transmitted and a reflected ray that are then both traced individually. Their respective power values are $P_{\text {trans }}=T P_{\mathrm{i}}$ and $P_{\text {refl }}=R P_{\mathrm{i}}$. Here, $T$ and $R$ are the transmittance and reflectance according to the Fresnel equations (for unpolarized light). This procedure is repeated in a recursive manner until every ray has left the simulation region. To calculate the relative improvement including all reflections, $\zeta^{\prime}$, it is insufficient to simply count the rays that are transmitted to the substrate (as in Equation (S9)), since they may carry different powers. Instead, we have to consider the total power transmitted to the substrate, $P^{\text {tot }}$. If we choose the same reference as in the calculation ignoring reflections, i.e. a silicon surface with contact grid, the relative improvement $\zeta_{\mathrm{Si}}^{\prime}$ becomes

$$
\zeta_{\mathrm{Si}}^{\prime}=\frac{P_{\mathrm{cloak}}^{\mathrm{tot}}}{T_{\mathrm{aS}} P_{0} N_{0}(1-f)}-1,
$$


where $T_{\mathrm{aS}}$ denotes the Fresnel transmittance from air to silicon. While this approach only requires one ray-tracing calculation to yield the relative improvement, it has a drawback: Since the invisibility structure exhibits a refractive index between that of silicon and that of air, it trivially reduces reflectivity of the whole arrangement. This effect is included in $\zeta_{\mathrm{Si}}^{\prime}$, which is not desirable. Instead, one should rather compare the performance of the invisibility structure to the performance of a different reference structure, e.g. a flat layer exhibiting the same refractive index as the invisibility structure. This, however, requires two ray-tracing simulation runs, to calculate the total transmitted power for both the invisibility structure, $P_{\text {cloak }}^{\text {tot }}$ and the reference structure, $P_{\text {ref }}^{\text {tot }}$. The relative improvement is then given as

$$
\zeta_{\text {ref }}^{\prime}=\frac{P_{\text {cloak }}^{\text {tot }}}{P_{\text {ref }}^{\text {tot }}}-1
$$

Its angular dependence for different types of invisibility structures is depicted in Figure S5. Firstly, it can be seen that the qualitative behavior in the most important angular range (below $40^{\circ}$ ) does not differ much from the calculation results without partial reflections (Figure 4 in the main text). The absolute value of the relative improvement, however, is different. This is due to the fact that the three types of invisibility structures exhibit different average refractive indices, and thus lead to different reductions of the total reflectivity of the overall structure. The optimum refractive index to reduce the overall reflectivity with a single additional layer is $n_{\mathrm{opt}}=\sqrt{n_{\mathrm{Si}}}=2$ (ignoring interference effects and multiple reflections). While the graded-index structures have an average refractive index of $n \approx 1.3$, that of the free-form surface structure is $n=1.5$, explaining the better anti-reflection effect of the latter. For the encapsulated free-form surface (see Section C), the reflectivity is reduced further, leading to an even higher value for $\zeta_{\text {flat }}^{\prime}$.

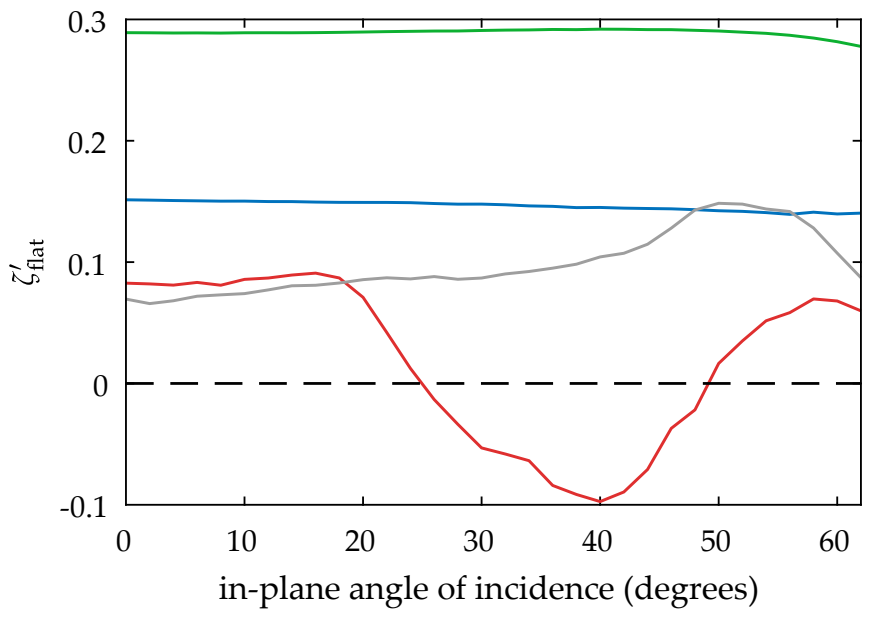

Fig. S5. Dependence of the calculated relative improvement on the in-plane incidence angle $\psi_{1}$, with $\psi_{2}=0^{\circ}$. Partial reflections are included for the graded-index structure (as depicted in Figure 1(b) of the main text) with polymer core (red), with air core (gray), the free-form surface (blue, as shown in Figure 2 of the main text) and the EVA-encapsulated $\mathrm{TiO}_{2}$ free-form surface (green). The relative improvement is referenced to a flat layer with homogeneous refractive index $n=1.5$ and thickness $R_{2}$ (dashed line).

It has to be noted that referencing the performance of the invisibility structure to something else than the silicon substrate also introduces slight changes to the computation of the annual average improvement. Assuming a constant number of rays $N_{0}$ (and constant initial power $P_{0}$ ) across the two simulation runs, the average relative improvement becomes

$$
\begin{aligned}
\left\langle\zeta_{\text {ref }}^{\prime}\right\rangle & =\frac{\text { energy deposited on active area with cloak }}{\text { energy deposited on active area with reference }}-1 \\
& =\frac{\int_{\text {year }} P_{\text {cloak }}^{\text {tot }}(t) I_{\text {dir }}(t)+\overline{T_{\text {cloak }}} I_{\text {diff }}(t) \mathrm{d} t}{\int_{\text {year }} P_{\text {ref }}^{\text {tot }}(t) I_{\text {dir }}(t)+\overline{T_{\text {ref }}} I_{\text {diff }}(t) \mathrm{d} t}-1 .
\end{aligned}
$$

Here, $\overline{T_{\text {ref }}}$ is the angle-averaged transmittance through the reference structure, i.e.,

$$
\overline{T_{\text {ref }}}=\frac{1}{2 \pi} \int_{0}^{2 \pi} \int_{0}^{\pi / 2}\left(\zeta_{\mathrm{ref}}(\vartheta, \varphi)+1\right) T_{\mathrm{aS}}(\vartheta) \sin \vartheta \mathrm{d} \vartheta \mathrm{d} \varphi,
$$

where $\zeta_{\text {ref }}(\vartheta, \varphi)$ denotes the improvement obtained by the reference structure relative to plain silicon. For reference, typical values of the annual average of the relative improvement for different invisibility structures, both ignoring and including partial reflections, are given in Table S1.

Table S1. Annual averages of relative improvement ignoring partial reflections, $\zeta$, and of relative improvement referenced to a flat layer with refractive index $n_{\text {flat }}=1.5$ and thickness $R_{2}$, including all reflections, $\zeta_{\text {flat }}^{\prime}$. Both quantities are given for contacts with areal filling fraction $f=0.1$ and width $w=R_{1} / 0.6$.

\begin{tabular}{ccc}
\hline invisibility structure & $\langle\zeta\rangle$ & $\left\langle\zeta_{\text {flat }}^{\prime}\right\rangle$ \\
\hline graded index (polymer core) & 0.04 & 0.07 \\
graded index (air core) & 0.09 & 0.10 \\
polymeric free-form surface & 0.11 & 0.20 \\
encapsulated $\mathrm{TiO}_{2}$ free-form surface & 0.11 & 0.29 \\
\hline
\end{tabular}

\section{Influence of an Encapsulant Layer}

Typically, real-world Si solar cells are encapsulated with an ethylene vinyl acetate (EVA) or silicone layer which is flat on the air-facing side. Characteristic refractive indices for such encapsulants are around $n \approx 1.5$ (Ref. [8]). Thus, a polymeric free-form structure as proposed in the main text would not lead to sufficient refraction of the incident light, as it exhibits a comparable refractive index. The functionality of the invisibility structure can be restored, however, by choosing a constituent material with a higher refractive index.

As an example, we run ray tracing simulations for a free-form surface made of $\mathrm{TiO}_{2}\left(n_{1}=2.7\right.$, Ref. [9] $)$ that sits on a silicon wafer and is encapsulated against air in a layer with refractive index $n_{0}=1.5$ and thickness $R_{2}$ (see Figure S6). Again, we assume an areal filling fraction $f=0.1$ of the contacts with width $w$. The geometric parameters of the free-form surface are chosen as $R_{1} / w=0.6, R_{2} / w=5$, and $y(0) / R_{1}=0.7$. It has to be noted that we substitute $n$ in Equations (S4) and (S5) by the ratio $n_{1} / n_{0}$ to compute the free-form surface function for the encapsulated invisibility structure.

Ignoring reflections the calculated relative improvement is $\zeta=0.11$ over the full in-plane angular range from $0^{\circ}$ to $70^{\circ}$, exactly as for the configuration without encapsulant layer. The 

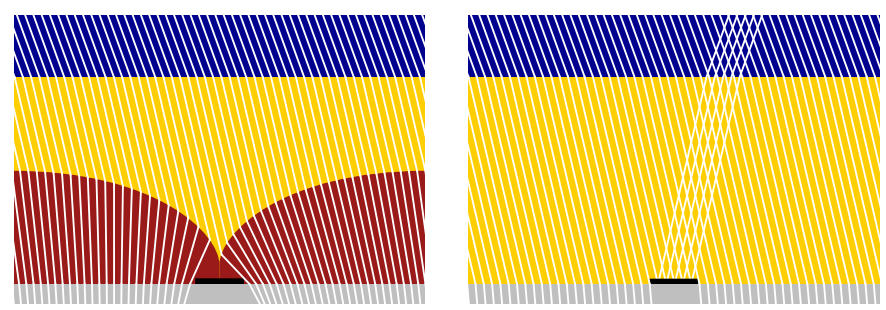

titanium dioxide, silicon dioxide, aluminum nitride, and silicon nitride," Applied Optics 51, 6789-6798 (2012).

Fig. S6. Refractive index maps (color-coded) with exemplary rays (white) as obtained by ray-tracing, showing the encapsulated free-form surface (left) and the corresponding reference structure (right). The refractive indices are chosen $n_{0}=1.5$ for the encapsulant material (yellow), $n_{1}=2.7$ for the constituent material of the free-form structure (red), and $n_{2}=4$ for the silicon substrate (gray). For clarity, partially reflected rays are not drawn. The rays are fully traveling in the $x y$-plane, i.e., $\psi_{2}=0^{\circ}$.

angular dependence of the relative improvement including partial reflections, $\zeta_{\text {flat }}^{\prime}$, is depicted in Figure S5. While showing a qualitatively similar behavior as the free-form surface at air, the absolute value of the relative improvement is higher for the encapsulated variant. This is due to the better anti-reflection effect provided by the two-layer structure. As all four curves are referenced to the same reference structure, namely a flat layer with refractive index $n=1.5$ and thickness $R_{2}$, the reduced reflectivity leads to a higher value of $\zeta_{\text {flat }}^{\prime}$.

\section{REFERENCES}

1. T. A. Driscoll and L. N. Trefethen, Schwarz-Christoffel Mapping (Cambridge University Press, 2002).

2. T. Baldacchini, C. N. LaFratta, R. A. Farrer, M. C. Teich, B. E. Saleh, M. J. Naughton, and J. T. Fourkas, "Acrylic-based resin with favorable properties for three-dimensional two-photon polymerization," Journal of Applied Physics 95, 6072-6076 (2004).

3. T. Ergin, N. Stenger, P. Brenner, J. B. Pendry, and M. Wegener, "Three-Dimensional Invisibility Cloak at Optical Wavelengths," Science 328, 337-339 (2010).

4. S. C. W. Krauter, Solar Electric Power Generation - Photovoltaic Energy Systems (Springer Berlin Heidelberg, 2006).

5. European Union, "PV potential estimation utility," http:/ / re.jrc.ec.europa.eu/pvgis/apps4/pvest.php, accessed 12/19/14.

6. T. Huld, R. Müller, and A. Gambardella, "A new solar radiation database for estimating PV performance in Europe and Africa," Solar Energy 86, 1803-1815 (2012).

7. J. C. Halimeh, R. Schmied, and M. Wegener, “Newtonian photorealistic ray tracing of grating cloaks and correlationfunction-based cloaking-quality assessment," Optics Express 19, 6078-6092 (2011).

8. K. McIntosh, J. Cotsell, J. Cumpston, A. Norris, N. Powell, and B. Ketola, "An optical comparison of silicone and EVA encapsulants for conventional silicon PV modules: A raytracing study," in "2009 34th IEEE Photovoltaic Specialists Conference (PVSC)," (2009), pp. 000544-000549.

9. J. Kischkat, S. Peters, B. Gruska, M. Semtsiv, M. Chashnikova, M. Klinkmüller, O. Fedosenko, S. Machulik, A. Aleksandrova, G. Monastyrskyi, Y. Flores, and W. Ted Masselink, "Midinfrared optical properties of thin films of aluminum oxide, 